\title{
Analysis of the Envelope Correlation Coefficient of MIMO Antennas Connected with Suspended Lines
}

\author{
Seung-Ho Kim · Jae-Young Chung*
}

\begin{abstract}
Inserting a suspended line is a widely used technique to reduce the envelope correlation coefficients (ECC) of multiple-input multipleoutput (MIMO) antennas, but its ECC reduction mechanism has not been carefully investigated so far. In this paper, MIMO antenna pairs connected with different suspended lines were evaluated using a full-wave simulation and measurement results. We calculated the surface current density at the center of the suspended line inserted between the antenna elements and found that the derivative of the surface current density was closely related to the ECC values. Furthermore, parametric studies showed that the suspended line length determines the ECC reduction bandwidth of the MIMO antenna, and the antenna length controls the ECC minimum frequency point. These guidelines provide useful insights into designing a low ECC MIMO antenna employing a suspended line.
\end{abstract}

Key Words: Envelope Correlation Coefficient, Microstrip Line, MIMO Antenna, Surface Current Density, Suspended Line.

\section{INTRODUCTION}

As information communication technology has been selected as the core growth engine of the fourth industrial revolution, there is a growing interest in constructing a network infrastructure with low latency and high connectivity. Fifth generation (5G) mobile communication is the technology on which such a network environment is based and is being actively implemented in several tech-leading countries. One of the key technologies employed not only in $4 \mathrm{G}$ but also in $5 \mathrm{G}$ is the multiple-input multiple-output (MIMO) antenna. This technology dramatically increases the data transmission rate without increasing the bandwidth or transmitting power by arranging multiple antennas at the transmitting and receiving ends $[1,2]$. In general, the performance of the MIMO antenna is strongly affected by the envelope correlation coefficient (ECC) between the antenna elements; a lower ECC offers the best performance [3]. The mutual coupling (MC) between the antennas significantly affects ECC. For example, the narrower the distance between the antenna elements, the stronger the mutual interference and the more fatal the effect on ECC and the overall MIMO system. To apply the MIMO antenna technology to a limited space (e.g., inside a cellphone), the $\mathrm{MC}$ and ECC problems must be solved simultaneously [4].

Many antenna structures and alignment techniques have been studied to solve this problem [5-10]. The most intuitive way to reduce the ECC of MIMO antennas is to increase space diversity or polarization diversity by widening the distance between the antenna elements or rotating the elements orthogonally to each other [5, 6]. However, this is not suitable for MIMO antennas implemented in a limited space. A defected ground structure (DGS) was proposed to reduce the ECC by changing the surface current distribution flowing on the ground plane [7, 8]. However, its use is limited because additional patterning on

Manuscript received September 15, 2019 ; Revised November 13, 2019 ; Accepted December 2, 2019. (ID No. 20190915-078J)

Department of Electrical and Information Engineering, Seoul National University of Science \& Technology, Seoul, Korea.

"Corresponding Author: Jae-Young Chung (e-mail: jychung@seoultech.ac.kr)

This is an Open-Access article distributed under the terms of the Creative Commons Attribution Non-Commercial License (http://creativecommons.org/licenses/by-nc/4.0) which permits unrestricted non-commercial use, distribution, and reproduction in any medium, provided the original work is properly cited.

(c) Copyright The Korean Institute of Electromagnetic Engineering and Science. All Rights Reserved. 
the ground is not preferred for the manufacturing viewpoint, and DGS patterns usually generate unwanted back lobes. Similarly, an electromagnetic band-gap (EBG) structure was proposed to reduce the ECC by suppressing surface waves on the ground plane $[9,10]$. However, the EBG structure complicates the antenna structure, and it is not suitable when the antenna elements are closely placed.

Inserting a suspended line between antenna elements is one promising way to reduce the ECC. The suspended line is known to effectively cancel MC between MIMO antennas, thereby reducing the ECC by generating a phase difference of $180^{\circ}$ from the interference signal to the signal flowing through the suspended line $[11,12]$. It is easy to implement not only for small terminals with limited space but also with various antenna structures. However, despite these advantages, there is no clear guideline for designing a suspended line for ECC reduction. Accordingly, many trial-and-error processes are required to find the optimal suspended line design. Only a few studies have analyzed the operation principle of the suspended line. In [4], an equivalent circuit of the suspended line was used to analyze it based on the relationship between impedance and surface current. In terms of the phase of the signal, one study described the operation principle of the suspended line based on a negative group delay phenomenon [13].

In this study, we analyzed the operation principle of a suspended line using full-wave simulations and MIMO antenna prototype measurements. Several MIMO antennas connected with suspended lines were modelled and fabricated, and their $S$ parameters, radiation patterns, ECC, and surface current densities were compared. We found that the reduction of ECC was strongly related to the derivative of the surface current magnitude in the middle of the suspended line, which supports the assumption of a $180^{\circ}$ out-of-phase cancellation in the surface currents.

\section{MIMO ANTENNA PROTOTYPE DESIGN}

\section{AND MEASUREMENT}

The geometries of the MIMO antenna models designed for the experiment are illustrated in Fig. 1. They are two-port MIMO antennas printed on the top side of an $87 \mathrm{~mm} \times 130$ $\mathrm{mm} \times 1.6 \mathrm{~mm}$ FR4 substrate. Two meander-line antennas are connected with a suspended line in the middle. The antenna models were designed to resonate at a frequency band of 0.8 $\mathrm{GHz}$. Otherwise, their ECC values were altered by adjusting the length of the suspended line. Accordingly, the high ECC model in Fig. 2(a) was designed to have an ECC of 0.8 or more, and the low ECC model in Fig. 2(b) was designed to have an ECC of 0.1 or less. A full-wave electromagnetic simulation software (i.e., Ansys HFSS v.18.1) was used to design the antenna models. Table 1 shows the geometrical parameters for the

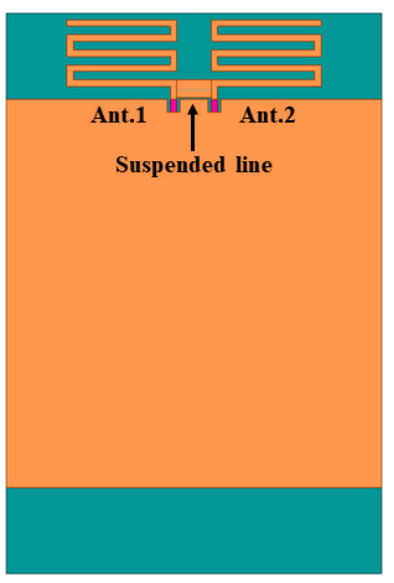

(a)

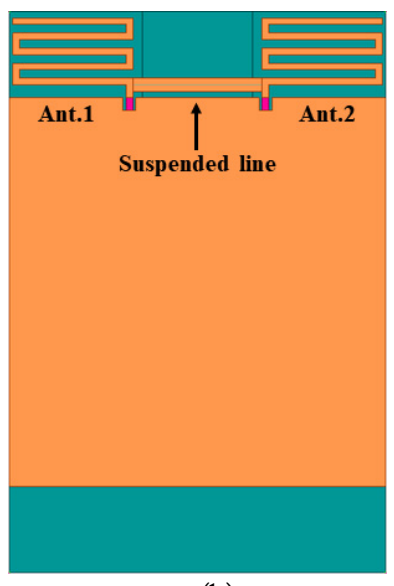

(b)
Fig. 1. Geometry of the designed MIMO antenna models: (a) high ECC model and (b) low ECC model.

\section{L1}

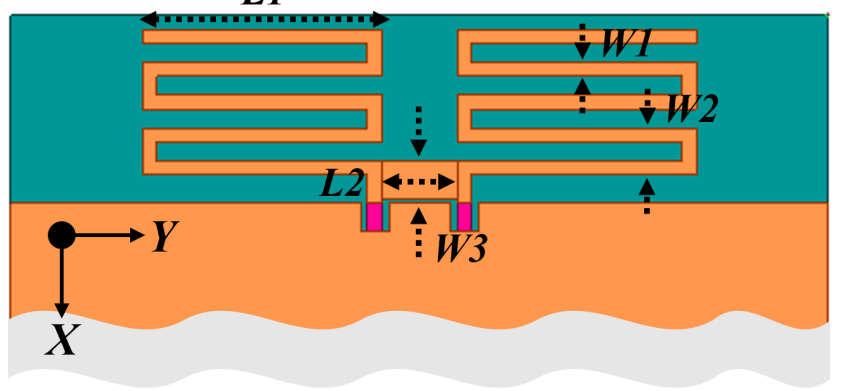

(a)

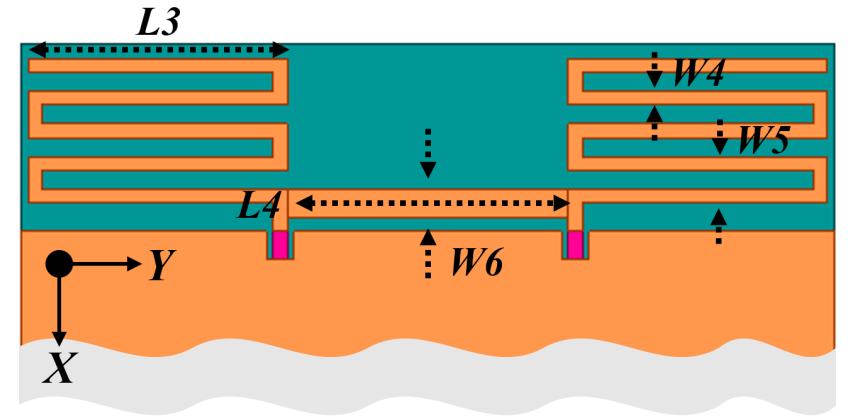

(b)

Fig. 2. Design parameters of the MIMO antenna models: (a) high ECC model and (b) low ECC model.

high and low ECC models. Based on these parameters, the prototype antennas fabricated by the printed circuit board (PCB) process are presented in Fig. 3. The ends of the meander antennas were further tuned to make the antennas resonate at 0.8 $\mathrm{GHz}$.

The reflection coefficient $\left(S_{11}\right)$ and the transmission coefficient $\left(S_{21}\right)$ of the prototype antennas were measured. Fig. 4 presents the simulated and measured $S_{i j}$ results. As shown in Fig. 4(a), the $S_{11}$ results of both the high and low ECC models are not like the conventional meander-line antenna with strong 
Table 1. Optimized antenna parameters (defined in Fig. 2)

\begin{tabular}{cc}
\hline Parameter & Value $(\mathrm{mm})$ \\
\hline$L_{1}$ & 25.5 \\
$L_{2}$ & 4 \\
$L_{3}$ & 27.7 \\
$L_{4}$ & 30 \\
$W_{1}$ & 1.5 \\
$W_{2}$ & 5 \\
$W_{3}$ & 4 \\
$W_{4}$ & 1.5 \\
$W_{5}$ & 5 \\
$W_{6}$ & 3 \\
\hline
\end{tabular}

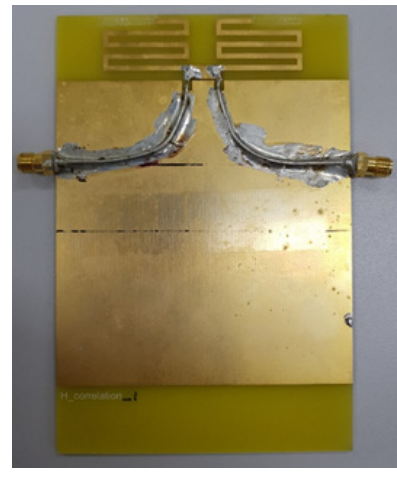

(a)

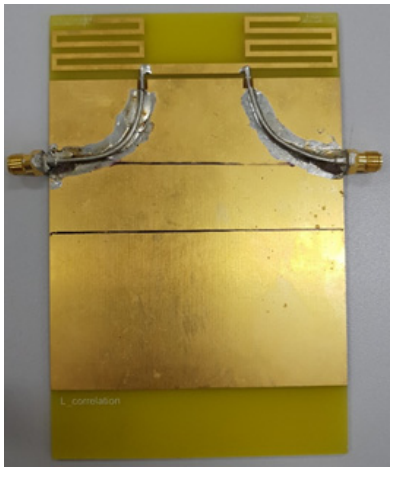

(b)
Fig. 3. Pictures of the fabricated prototype antennas fed by $50 \Omega$ coaxial cables: (a) high ECC model and (b) low ECC model.

resonance. They act like broadband antennas, as the two meander lines are bridged by the suspended line. The $S_{11}$ magnitude increases as the frequency increases, with a slight dip around the operation frequency $(0.8 \mathrm{GHz})$. The simulation and measurement results show good agreement. The slight deviation may result from possible fabrication errors, particularly for the $S_{11}$ graphs, which have a relatively lower level. Nevertheless, the $S_{11}$ magnitude values at $0.8 \mathrm{GHz}$ are all less than $-9 \mathrm{~dB}$. Conversely, the $S_{21}$ results in Fig. 4(b) show a good agreement between the simulation and the measured results. Note that $S_{21}$ of the low ECC model shows a sharp resonance-like valley at $0.8 \mathrm{GHz}$, but the high ECC model maintains a high $S_{21}$ value. $S_{21}$ implies the mutual coupling between two antennas. Thus, ECC is predicted to be low and high for the low and high ECC models, respectively.

We then measured the far-field radiation patterns of the prototype antennas to calculate the ECC values. For the ECC calculation, although a method using $S$-parameters as in (1) can be employed, this method is effective only for an antenna with a high-radiation efficiency, and it is not suitable for calculating the ECC between small antennas mounted on the terminal [14].

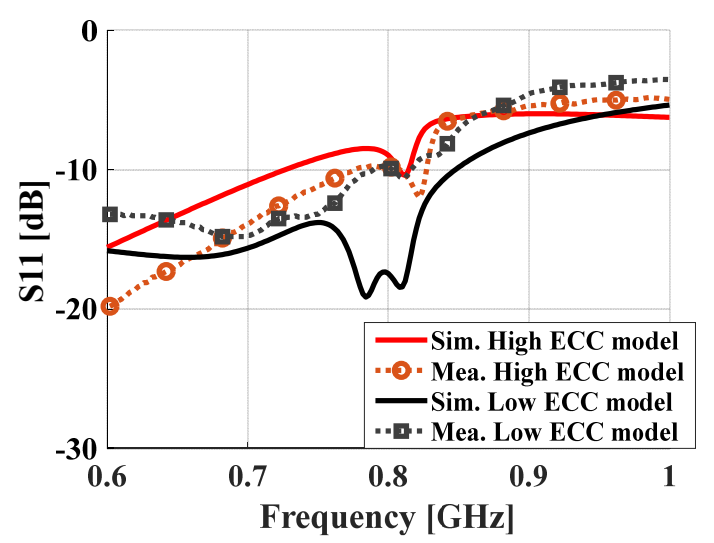

(a)

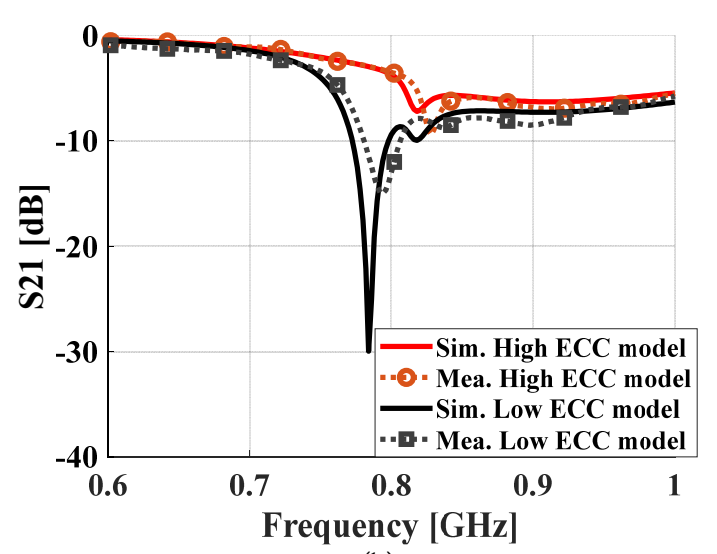

(b)

Fig. 4. Comparisons of the $S$-parameter results between the simulations and measurements of the prototype antennas: (a) $S_{11}$ and (b) $S_{12}$ results.

$$
\rho_{12}=\frac{\left|S_{11}^{*} S_{12}+S_{12}^{*} S_{22}\right|^{2}}{\left(1-\left|S_{11}\right|^{2}-\left|S_{21}\right|^{2}\right)\left(1-\left|S_{22}\right|^{2}-\left|S_{12}\right|^{2}\right)}
$$

Instead, we calculated the ECC using 3D radiation patterns measured in an electromagnetic anechoic chamber. The equation used is as follows:

$$
|\boldsymbol{\rho}|=\frac{\left|\oint \boldsymbol{E}_{\mathbf{1}} \cdot \boldsymbol{E}_{2}^{*} d \Omega\right|^{2}}{\left|\oint \boldsymbol{E}_{\mathbf{1}} \cdot \boldsymbol{E}_{\mathbf{1}}^{*} d \Omega \times \oint \boldsymbol{E}_{2} \cdot \boldsymbol{E}_{2}^{*} d \Omega\right|}
$$

where $\Omega=\sin \theta d \theta d \phi$ is the beam solid angle, and $E$ is the electric fields received at the measurement probe in the far-field range. The subscript indicates the electric fields received from antenna 1 or 2 . Fig. 5 shows the anechoic chamber setup for the measurements.

Fig. 6 presents the ECC values computed using the simulated and measured 3D radiation patterns. The slight deviations between the simulation and measurement could have originated from the difference between the chamber testing environment and the ideal simulation.

For the high ECC model (Fig. 6(a)), ECC is low at 0.82 $\mathrm{GHz}$ but high at $0.8 \mathrm{GHz}$ as intended. The measured ECC 


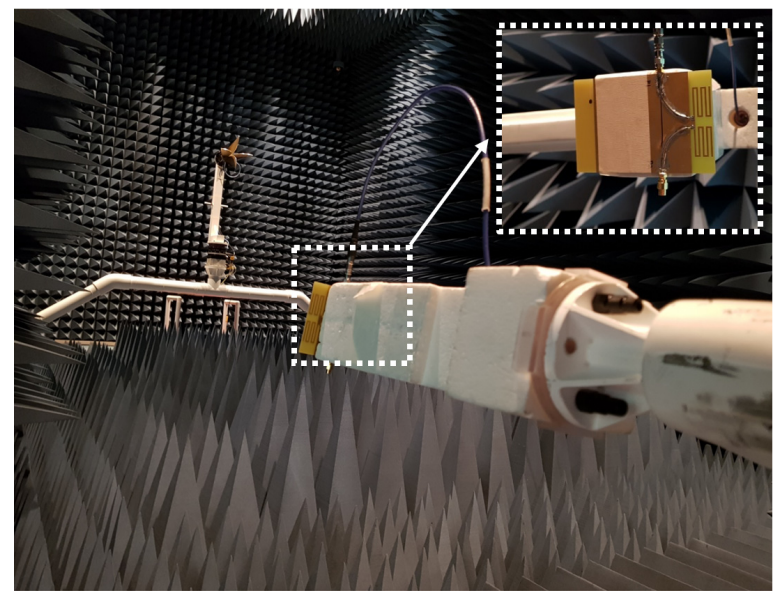

Fig. 5. Measurement setup for the far-field radiation characteristics of the prototype antennas in an electromagnetic anechoic chamber.

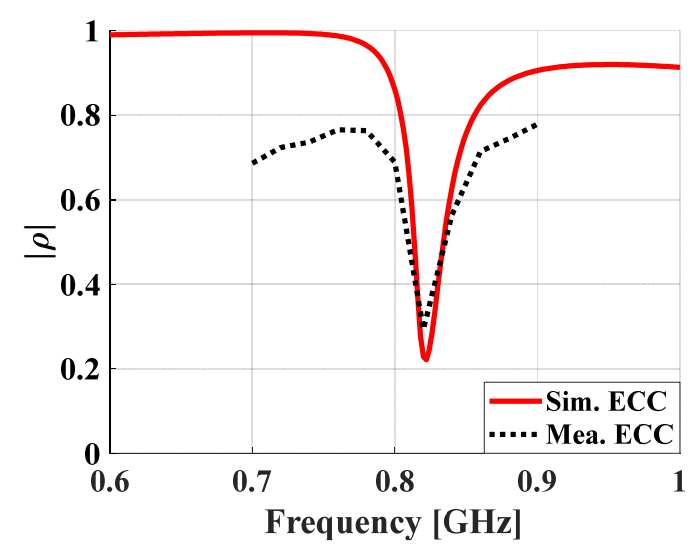

(a)

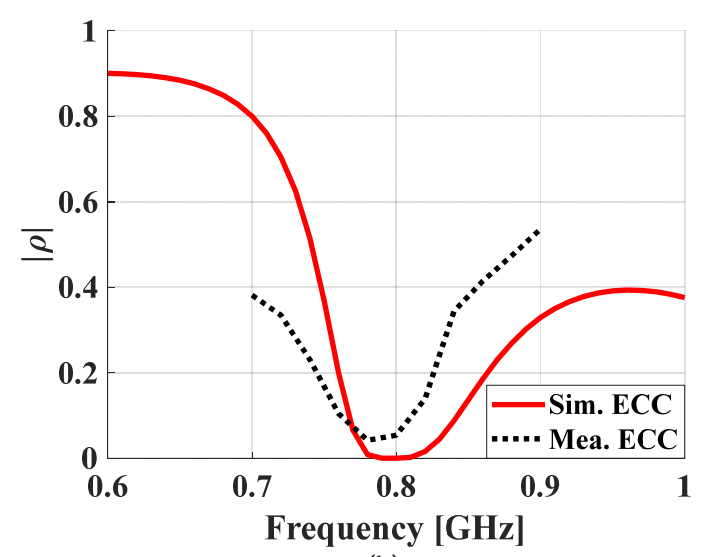

(b)

Fig. 6. Comparison of the ECC between the simulation and measurement results of the prototype antenna: (a) high ECC model and (b) low ECC model.

value at $0.8 \mathrm{GHz}$ is 0.69 for the low ECC model (Fig. 6(b)). At $0.8 \mathrm{GHz}$, the measured $\mathrm{ECC}$ value at $0.8 \mathrm{GHz}$ is only 0.076 and shows a broader frequency range exhibiting the null.

\section{ANALYSIS OF THE ECC REDUCTION EFFECT BYTHE SUSPENDED LINE}

Having verified that the full-wave simulation results and measured results showed similar tendencies, we analyzed the ECC reduction effect by calculating the surface current densities $\left(J_{\text {surf }}\right)$ on the suspended line. For this purpose, the observation points for calculating $J_{\text {surf }}$ on the suspended line of the high/low ECC model were set up. As shown in Fig. 7(a) and (b), the center of the suspended line was chosen for the high and low ECC models, respectively.

Fig. 8(a) shows the calculated magnitude of $J_{\text {surf }}$ at the center point 1 for the high and low ECC models. It is interesting to note the abrupt changes at the lowest ECC frequency. The null of the ECC of the two models was located at the point where the magnitude of the surface current density changed sharply. On this basis, we can confirm that the two indicators have a certain relationship. To further analyze the relationship between $J_{\text {surf }}$ and ECC, the magnitude of $J_{\text {surf }}$ was differentiated with respect to the frequency and then compared with the ECC curves, as presented in Fig. 8(b).

The differentiated response of $J_{\text {surf }}$ showed a phenomenon in which the value rapidly decreased at the minimum ECC frequency, while a constant value was maintained. This result supports the cancellation of the surface currents flowing from one antenna to another due to a $180^{\circ}$ out-of-phase condition as the frequency varies.

For the high ECC model, the $180^{\circ}$ out-of-phase frequency

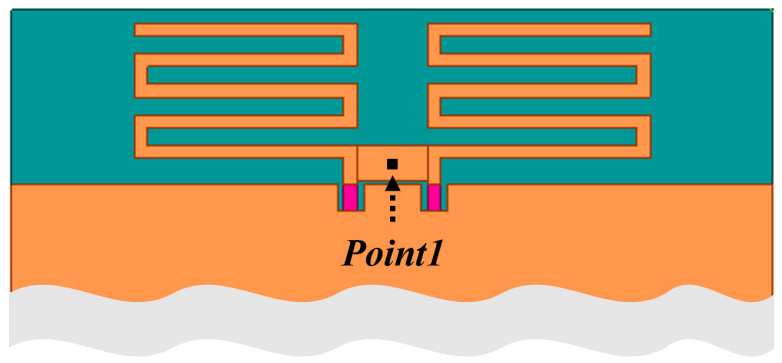

(a)

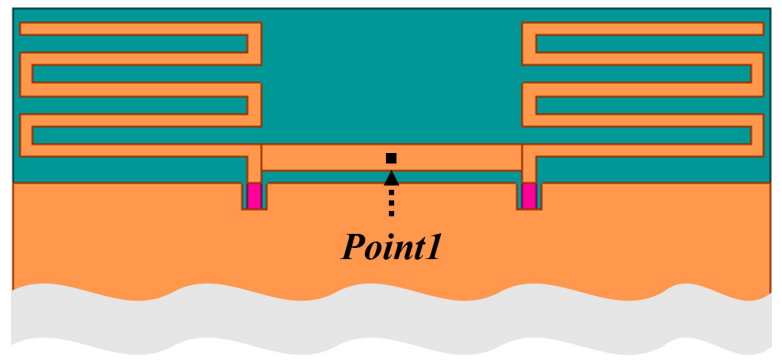

(b)

Fig. 7. Surface current density calculation point for the (a) high ECC model and (b) low ECC model. 


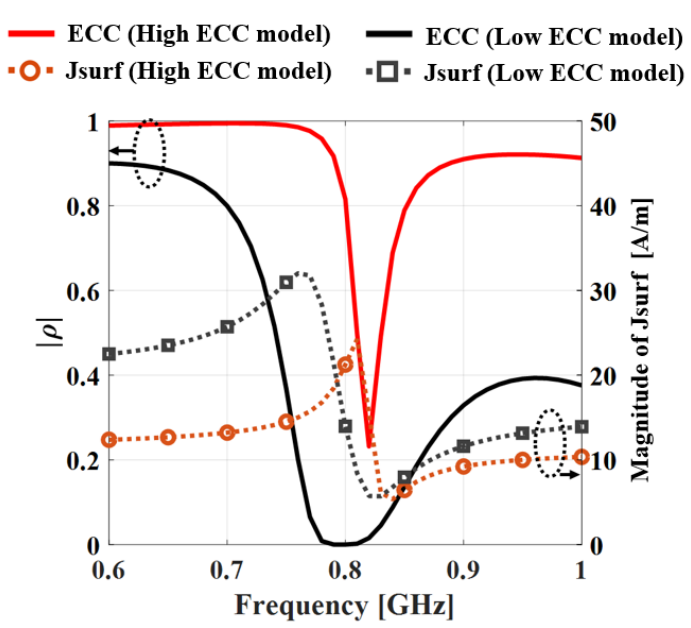

(a)

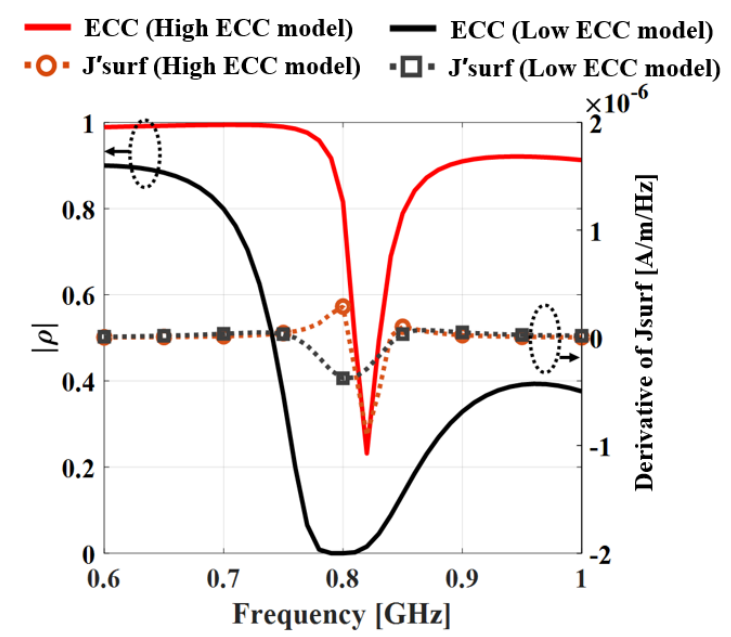

(b)

Fig. 8. Comparison of the ECC and $J_{\text {surf }}$ calculated at point 1: (a) ECC and magnitude of $J_{\text {surf }}$ and (b) ECC and derivative of $J_{\text {surf }}$.

band (i.e., $J_{\text {surf }}$ abruptly changing band) is narrow and located next to the frequency of interest $(0.8 \mathrm{GHz})$. Therefore, the ECC at $0.8 \mathrm{GHz}$ is high. Conversely, the $180^{\circ}$ out-of-phase band of the low ECC model is smooth and broad, resulting in a broader low ECC frequency response.

We performed a parametric study to further investigate the relationship between the ECC and the derivative of $J_{\text {surf }}$. Specifically, the length of the suspended line and the length of the antenna, which greatly affect the MIMO antenna's ECC, were set as the design parameters, as shown in Fig. 9. Accordingly, $L$ was set as the variable for adjusting the length of the antenna, and $Y Y$ was set as the variable for adjusting the length of the suspended line. As the width of the antenna and the thickness of the suspended line had no significant effect, they were not considered as design parameters for the parametric study.

Fig. 10 presents the parametric study results of the high ECC model. For the parametric study, the $L$ and $Y Y$ values of the

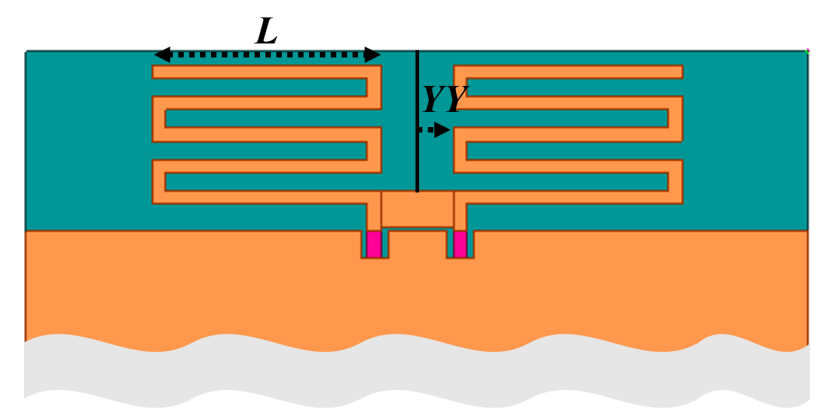

(a)

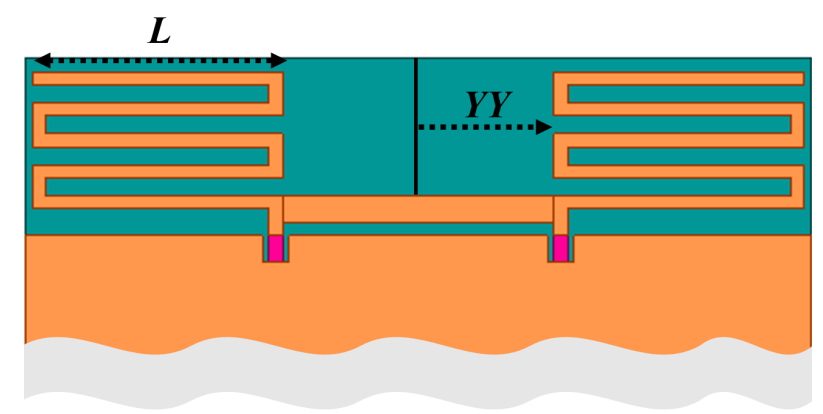

(b)

Fig. 9. Design parameters for parametric studies: (a) high ECC model and (b) low ECC model.

high ECC model were varied from $25.5 \mathrm{~mm}$ to $29.5 \mathrm{~mm}$ and from $5.5 \mathrm{~mm}$ to $9.5 \mathrm{~mm}$, respectively, and the resulting variation profiles for the ECC and the derivative of $J_{\text {surf }}$ were shown. Fig. 10(a), (b), and (c) illustrate the variation of the ECC and the derivative of $J_{\text {surf }}$ according to the change in the design variable $L$. As the $L$ value increases, the null of the two indicators shifts to the low-frequency band, similar to the decrease in antenna resonant frequency shifts as the antenna length increases.

Fig. 10(d), (e), and (f) illustrate the variation in the ECC and the derivative of $J_{\text {surf }}$ according to the change in the design variable $Y Y$. As $Y Y$ increases, the bandwidth around the null of the ECC widens from $50 \mathrm{MHz}$ to $70 \mathrm{MHz}$. This phenomenon occurred because the impedance of the suspended line decreased as the $Y Y$ value increased, and accordingly, the amount of the surface current flowing through the suspended line changed. Considering the above, the ECC minimum frequency and bandwidth can be controlled by $L$ and $Y Y$, which are the length of the antenna and suspended line, respectively.

In the same way, we performed a parametric study on the low ECC model, and the results are presented in Fig. 11. For the parametric study of the low ECC model, the $L$ and $Y Y$ values were varied from $23.7 \mathrm{~mm}$ to $27.7 \mathrm{~mm}$ and from $12.5 \mathrm{~mm}$ to $16.5 \mathrm{~mm}$, respectively. Fig. 11(a), (b), and (c) illustrate the variation of the ECC and the derivative of $J_{\text {surf according to the }}$ change in $L$. Similar to the high ECC model, as the $L$ value increases, the null of the two indicators shifts together to the low-frequency band. 


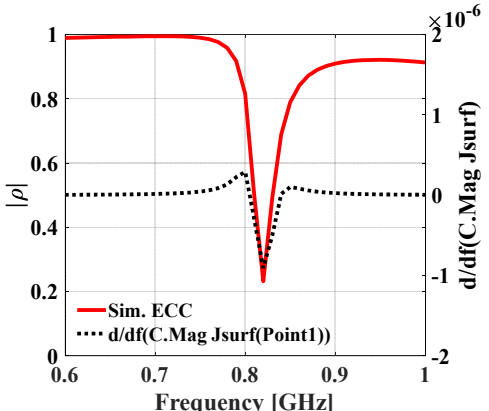

(a)

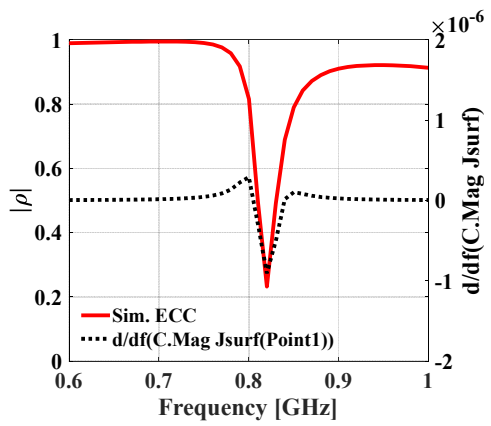

(d)

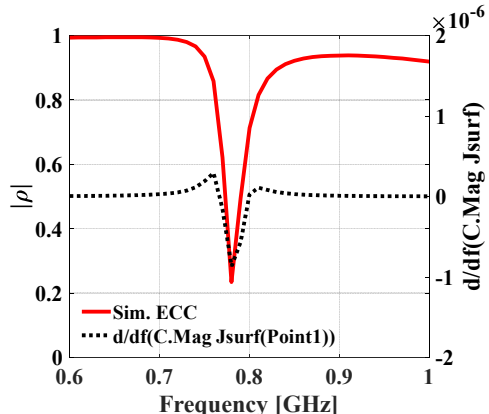

(b)

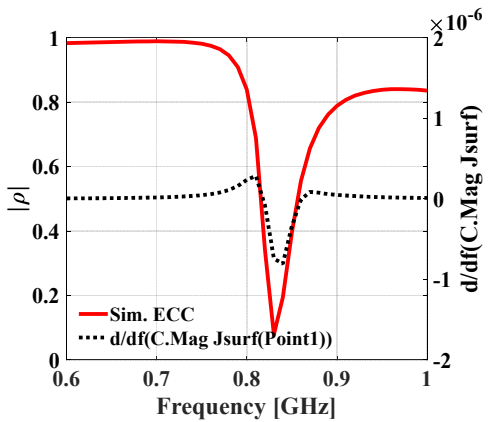

(e)

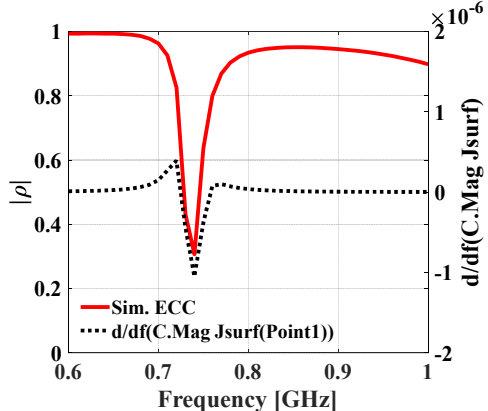

(c)

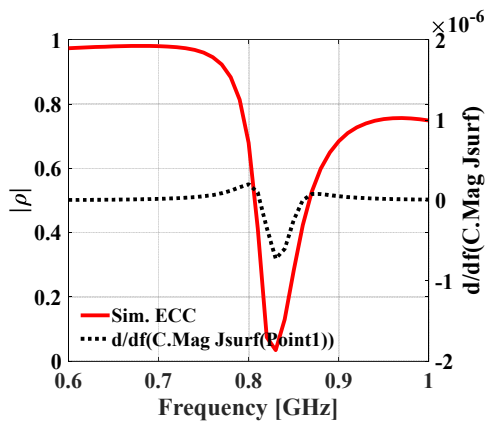

(f)

Fig. 10. Variation of the ECC and the derivative of $J_{\text {surf }}$ according to the change in design parameters of the high ECC model. (a) $L=$ $25.5 \mathrm{~mm}$, (b) $L=27.5 \mathrm{~mm}$, (c) $L=29.5 \mathrm{~mm}$, (d) $Y Y=5.5 \mathrm{~mm}$, (e) $Y Y=7.5 \mathrm{~mm}$, (f) $Y Y=9.5 \mathrm{~mm}$.

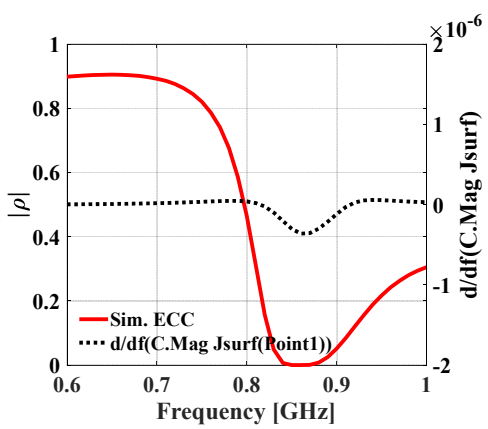

(a)

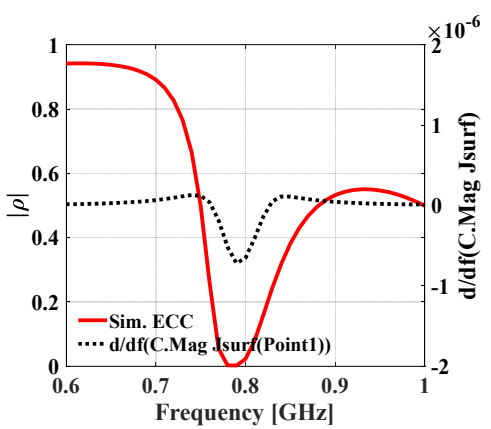

(d)

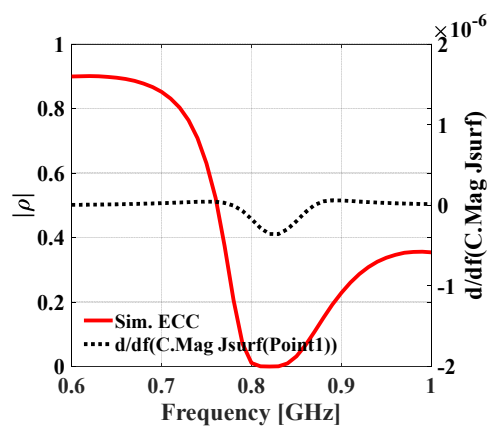

(b)

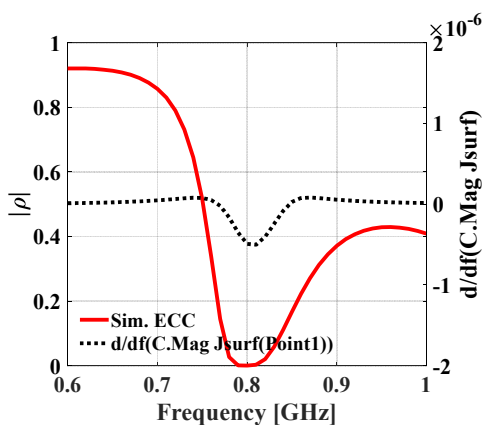

(e)

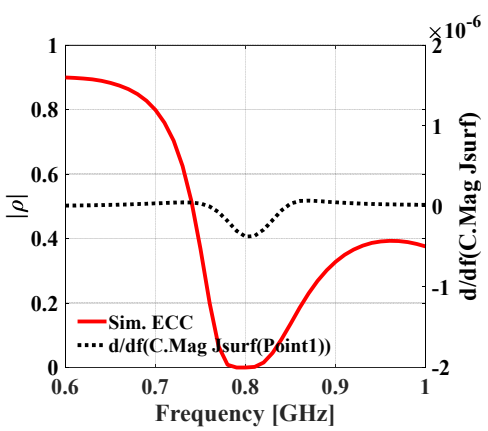

(c)

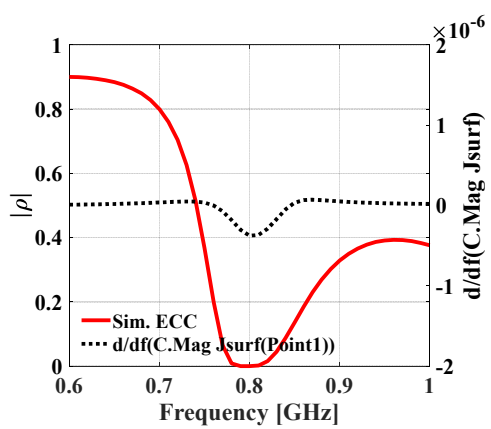

(f)

Fig. 11. Variation of the ECC and the surface current, which is differentiated in terms of frequency according to the change in the design parameters of the low ECC model. (a) $L=23.7 \mathrm{~mm}$, (b) $L=25.7 \mathrm{~mm}$, (c) $L=27.7 \mathrm{~mm}$, (d) $Y Y=12.5 \mathrm{~mm}$, (e) $Y Y=14.5 \mathrm{~mm}$, (f) $Y Y=16.5 \mathrm{~mm}$.

Fig. 11(d), (e), and (f) show the variation of the ECC and the derivative of $J_{\text {surf }}$ according to the change in $Y Y$. As $Y Y$ increases, the bandwidth around the null of the two indicators widens from $100 \mathrm{MHz}$ to $140 \mathrm{MHz}$. From the parametric study results of the two models for the design variable $Y Y$, the length of the suspended line determines the low ECC band- 
width.

\section{CONCLUSION}

In this paper, we analyzed the relationship between ECC, $J_{\text {surf }}$, and its derivative of a MIMO antenna connected with a suspended line. A pair of two-port MIMO antenna models exhibiting high and low ECC values were designed by inserting a short and long suspended line between the antenna elements. For each model, the antenna prototype was fabricated, and its $S$-parameters and radiation patterns were measured. With the measured 3D radiation patterns, the ECC was calculated and compared with the simulation results. The ECC reduction effect could be clearly observed in different ways for the high and low ECC models. The high ECC model showed an abrupt change in ECC along the frequency, whereas the low ECC model showed a relatively smooth change in ECC, resulting in a wider ECC minimum bandwidth.

These abrupt and smooth variations along the frequency turned out to be related to the profile of the $J_{\text {surf }}$ magnitude and its derivative in terms of frequency. This dispersive phenomenon is similar to the group delay, which is the phase derivative in terms of frequency. In fact, the ECC reduction effect due to the suspended line can be explained by the negative group delay phenomenon with a circuit, including lossy loads [13]. The latter can be considered as the MIMO antennas with high-radiation losses for the current study. By adding the suspended line, which is low loss, between these lossy antennas, the overall circuit becomes highly dispersive, and the group delay between the two ports exhibits a negative sign in a limited frequency range (i.e., the phase of $S_{21}$ increases as the frequency increases).

To further investigate the relationship between the ECC and the derivative of $J_{\text {surf }}$, the changes in these two indicators were observed by varying the length of the MIMO antenna and that of the suspended line. The results show that the ECC minimum frequency can be adjusted by the antenna length and that the ECC minimum frequency bandwidth is controllable by the suspended line length. We believe that these results can provide meaningful insights for MIMO antenna designers in the field.

This study was supported by the research program funded by Seoul National University of Science and Technology (Seoulech).

\section{REFERENCES}

[1] B. M. Hochwald and S. Ten Brink, "Achieving near-capacity on a multiple-antenna channel," IEEE Transactions on Co- mmunications, vol. 51, no. 3, pp. 389-399, 2003.

[2] H. Sampath, S. Talwar, J. Tellado, V. Erceg, and A. Paulraj, "A fourth-generation MIMO-OFDM broadband wireless system: design, performance, and field trial results," IEEE Communications Magazine, vol. 40, no. 9, pp. 143-149, 2002.

[3] Y. Yu, L. Yi, X. Liu, and Z. Gu, "Mutual coupling reduction of dual-frequency MIMO array with neutralization lines," in Proceedings of 2015 Asia-Pacific Microwave Conference (APMC), Nanjing, China, 2015, pp. 1-3.

[4] Y. Wang and Z. Du, "A wideband printed dual-antenna system with a novel neutralization line for mobile terminals," IEEE Antennas and Wireless Propagation Letters, vol. 12, pp. 1428-1431, 2013.

[5] J. Motohashi and M. Yamamoto, "A wideband 4-port MIMO antenna using leaf-shaped notch antennas," in Proceedings of 2016 International Symposium on Antennas and Propagation (ISAP), Okinawa, Japan, 2016, pp. 1070-1071.

[6] M. Karaboikis, C. Soras, G. Tsachtsiris, V. Papamichael, and V.Makios, "Multi element antenna systems for diversity and MIMO terminal devices," in PIERS 2004: Progress in Electromagnetics Research Symposium, Pisa, Italy, 2004.

[7] D. B. Lin, P. W. Lin, and C. Y. Chang, "Design for Coplanar MIMO LTE antenna in handset device application," in Proceedings of 2015 International Workshop on Electromagnetics: Applications and Student Innovation Competition (iWEM), Hsinchu, Taiwan, 2015, pp. 1-2.

[8] Y. Chung, S. S. Jeon, D. Ahn, J. I. Choi, and T. Itoh, "High isolation dual-polarized patch antenna using integrated defected ground structure," IEEE Microwave and Wireless Components Letters, vol. 14, no. 1, pp. 4-6, 2004.

[9] S. S. Alja' afreh, Y. Huang, Q.Xu, L.Xing, and O. A. Saaereh, "MIMO antenna system of a compact 4-element PILA for 4G handset applications," in Proceedings of 2016 Loughborough Antennas \& Propagation Conference (LAPC), Loughborough, UK, 2016, pp. 1-4.

[10] D. M. N. Elsheakh, M. F. Iskander, E. A. Abdallah, H. A. Elsadek, and H. Elhenawy, "Microstrip array antenna with new 2D-electromagnetic band gap structure shapes to reduce harmonics and mutual coupling," Progress In Electromagnetics Research, vol. 12, pp. 203-213, 2010.

[11] Y. Wang and Z. Du, "A wideband printed dual-antenna with three neutralization lines for mobile terminals," IEEE Transactions on Antennas and Propagation, vol. 62, no. 3, pp. 1495-1500, 2013.

[12] A. Diallo, C. Luxey, P. Le Thuc, R. Staraj, and G. Kossiavas, "Study and reduction of the mutual coupling between two mobile phone PIFAs operating in the DCS1800 and UMTS bands," IEEE Transactions on Antennas and Propagation, vol. 54, no. 11, pp. 3063-3074, 2006.

[13] J. Y. Chung, T. Yang, and J. Lee, "Low correlation MIMO 
antennas with negative group delay," Progress In Electromagnetics Research, vol. 22, pp. 151-163, 2011.

[14] A. Diallo, C. Luxey, P. Le Thuc, R. Staraj, and G. Kossiavas, "Enhanced two-antenna structures for universal mobile

\section{Seung-Ho Kim}

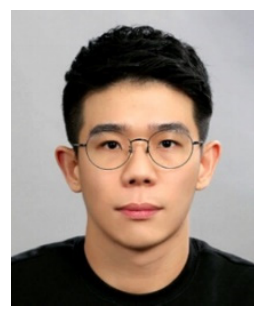

received his B.S. and M.S. degrees from the Department of Electrical and Information Engineering, Seoul National University of Science and Technology in 2017 and 2019, respectively. He currently works at Hyundai Motor Company. His research interests include antenna and RF circuit designs. telecommunications system diversity terminals," IET Microwaves, Antennas \& Propagation, vol. 2, no. 1, pp. 93-101, 2008.

Jae-Young Chung

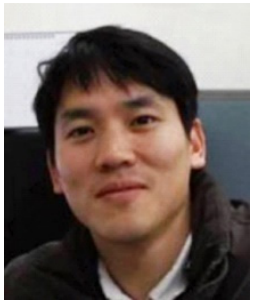

received his B.S. degree from Yonsei University, Seoul, South Korea, in 2002, and his M.S. and $\mathrm{Ph}$.D. degrees from the Ohio State University, Columbus, OH, USA, in 2007 and 2010, respectively, all in electrical engineering. From 2002 to 2004, he was an RF engineer in Motorola Korea. From 2010 to 2012, he was an antenna engineer in Samsung Electronics. He is currently an associate professor at the Department of Electrical and Information Engineering, Seoul National University of Science and Technology. His research interests include electromagnetic measurement and antenna design. 